\title{
LASER-BASED SECONDARY NEUTRAL MASS SPECTROSCOPY: USEFUL YIELD AND SENSITIVITY*
}

CONF-860807--4

DE87 011464
C. E. Young, M. J. Pellin, W. F. Calaway, B. Jørgensen, ${ }^{* *}$ E. L. Schweitzer, and D. M. Gruen Materials Science and Chemistry Divisions Argonne National Laboratory Argonne, IL 60439
BQB?

UUL $0: 3 \%$

Multiphoton ionization (MPl) by pulsed, tunable lasers provides a sensitive means for detection of neutral atoms due to the high efficiency achievable both in the ionization and subsequent detection. Substantial selectivity can be achieved by excitation between energy levels of the atom of interest. This rescnant MPI technique can access all atomic states of any particular atom including its ground and metastable levels. In principle all elements may be ionized through judicious selection of the color of the excitation laser light. In practice resonance ionization has been experimentally demonstrated for nearly every element .

A variety of problems exist in order to optimally apply resonance ionization spectroscopy (RIS) to the detection of sputtered neutral atoms, however. Several of these problems and their solutions are examined in this paper. First, the possible useful yields obtainable and the dependence of useful yield on various laser parameters for this type of sputtered neutral mass spectrometer (SNMS) are considered. Second, the choice of a mass spectrometer and its efiect on the instrumental useful yield is explored in light of the unique ionization region for laser based SNMS. Finally a briaf description of noise sources and their effect on the instrumental sensitivity is discussed.

That it is possible to combine in one instrument both high useful yields and high sensitivity for the detection of minority species (either very dilute surface constituents or species sputtered in highly excited states) will be demonstated with results on Fe implanted Si samples in the surface analysis by resonant ionization of sputtered atoms (SARISA) instrument. SARISA acomplishes the necessary noise reduction without signal loss through the extraction of the photoions into a sector-field time-of-flight (TOF) mass spectrometer. In standard, isochronous operation, energy and angular spreads at the point of ionization are compensated in flight to produce wellresolved TOF mass spectra. Noise sources (photons, metastable and scattered atoms) escaping through transparent grids are strongly suppressed.

\footnotetext{
*Work supported by the U.S. Department of Energy, BES-Materials Science, under Contract W-31-109-ENG-38.

${ }^{\star \star}$ Fysisk Institut, Odense Universitet, Denmark.
} 


\section{DISCLAIMER}

This report was prepared as an account of work sponsored by an agency of the United States Government. Neither the United States Government nor any agency thereof, nor any of their employees. makes any warranty, express or implied, or assumes any legal liability or responsibility for the accuracy, completeness, or usefulness of any information, apparatus, product, or process disclosed, or represents that its use would not infringe privately owned rights. Ruference herein to any specific commercial product, process, or service by trade name, trademark. manufacturer. or otherwise does not necessarily constitute or imply its endorsement, recommendation, or favoring by the United States Government or any agency thereof. The views and opinions of authors expressed herein do not necessarily state or reflect those of the United States Government or any agency thereof. 


\section{Introduction}

The past several years has seen a rapid development in laser-related surface measurement techniques. In particular, laser multiphoton ionization (MPI) of sputtered atoms has proven to be a sensitive probe, both of the sputtering process [1,2] and of surface composition [3-7]. This laser ionization version of secondary neutral mass spectrometry (LSNMS) has a threefold advantage in surface analysis when compared to Secondary lon Mass Spectrometry (SIMS). First, laser ionization techniques measure the dominant fraction of the sputtered flux (for metals and semiconductors [8]) - neutral atoms. Second, because the dominant neutral portion of the sputtered flux is measured, large changes in signal due to minor surface chemical effects may be minimized [9]. However, in cases where neutral atoms do not represent the dominant portion of the sputtered flux, such as when oxygen is absorbed on the surface in the presence of extended sputtering [10] or for oxide materials [7], this may not be the case. Finally in the case of Resonance lonization Spectroscopy (RIS), the laser ionization process is so species specific [11] that the stringent requirements for high mass resolution spectrometers are strongly alleviated making it possible to achieve much better transmission.

A comparison of LSNMS to other sputtered neutral mass spectrometric (SNMS) techniques such as the plasma ionization method of Oechsner [12] has been drawn previously [13] based on their relative ionization efficiency, the range of species ionized, and the experimental duty cycle. In this paper, a more detailed comparison of the various LSNMS techniques will be made and a few of the most exciting new results will be reviewed.

Basically, LSNMS represents an extremely efficient, though low-duty factor, ionization method. For sufficiently large laser powers, most elements may be ionized with unit efficiency. This can be compared to electron ionization effi ciencies if $\sim 0.01 \%$ [14] and hot electron gas ionization efficiencies of $1 \%$ [12]. For most SNMS techniques the ionization efficiency is reasonably mass independent [13]. For LSNMS the ionization can be species unspecific (nonresonant) or very species specific (resonant). Finally, one must consider the duty cycle of the experiment. Most SNMS techniques operate with unit duty factor, while this is not the case with LSNMS. The LSNMS duty factor is limited by the laser repetition rate of commercially available pulsed lasers to $10^{-4}$ - a significant disadvantage for routine analysis.

The discussion of LSNMS techniques in the present paper will focus on two important quantities for surface analysis - the useful yield, $\psi$, and the bulk sensitivity limit. The useful yield is defined as

$$
\psi=\text { atoms detected/atoms sputtered }
$$

This important quantity represents the key limit for micro characterization of samples with atomic dimensions, e. g., surface monolayers. 
The bulk sensitivity limit of a technique represents a different type of sensi tivity. In the overall context of materials analysis, it might be better character ized as near surface analysis. Several factors play a role in this limit. In the case of duty cycle limited experiments such as LSNMS, there is essentially no difference between sample limited (surface) analysis and near surface analy sis. For the more conventional SNMS techniques [12,14] and for SIMS duty cycle is not a limiting problem. In these cases the bulk sensitivity limit is a function of the signal-to-noise ratio of the measurement without regard to sample consumption. The signal-to-noise ratio will be enhanced for succes sively longer averaging times until the bulk sensitivity limit is reached. Often this limit is due to an isobaric interference. In the best cases this limit arises from detector dark current.

In this paper, three laser ionization surface analytical instruments will be compared and contrasted. In this rapidly advancing field, it is impossible to consider in detail all of the excellent work which is being done and the reader is asked to take this circumstance into account. Rather than an exhaustive survey, we endeavor instead to produce an illustrative review which will allow the reader quick entry into the field. The three instruments that are detailed are the sputter-initiated resonance ionization spectroscopy (SIRIS) instrument [5], the surface analysis by resonance ionization of sputtered atoms (SARISA) instrument [4] and the surface analysis by laser ionization (SALI) instrument [3].

\section{Experimental}

Figures 1-3 present the three experimental approaches which will be discussed in some detail in this paper. The drawings are schematic in nature and are intended to be illustrative of the underlying principles which each instrument embodies. Let us examine each of these in turn.

Figure 1 is a generic schematic of the type of SALI apparatus that has been used by Becker and Gillen [3] to nonresonantly ionize sputtered atoms and then to analyze the photoionized flux by time-of-flight (TOF) mass spectrometry. The system is contained in a UHV apparatus with a base pressure [3] of $10^{-9}$ mbar. The timing diagram of fig. 4 should be consulted in an examination of this apparatus. Those time lines with the label 1 are used in the SALI apparatus. The experimental sequence of fig. 1 is initiated by an intense nonresonant laser pulse which ionizes the sputtered flux produced by a pulsed $\mathrm{Ar}^{+}$(or other primary ion) beam ( $2 \mathrm{keV}$ ). The laser pulse itself passes $1 \mathrm{~mm}$ in front of the target surface and is focused to a $0.2 \mathrm{~mm}$ waist. The ionizing radiation is usually ultraviolet light (in general of wavelength $193 \mathrm{~nm}$ or $248 \mathrm{~nm}$ ) with an intensity in the range $10^{8}$ to $10^{12} \mathrm{~W} / \mathrm{cm}^{2}$. The photoions created are then extracted into a TOF mass analyzer of the reflectron type [15].

Figure 2 is a schematic of an apparatus after Parks et al. [5] called SIRIS. Referring to the time lines of fig. 4 which are labeled 2 , one finds that the 
experimental sequence of SIRIS is initiated by a pulse of 5 to $30 \mathrm{keV} \mathrm{Ar+} \mathrm{(or}$ other primary ion) ions. This pulse is typically $0.75 \mu \mathrm{sec}$ long with a peak current of $50 \mu \mathrm{amp}$. The sputtered flux contains both secondary neutral atoms and secondary ions. The neutral atoms of the element to be studied are then ionized in a resonant ionization scheme [5] using two- and sometimes threetime coincident laser pulses. The laser light is focused to a $1 \mathrm{~mm}$ dia. spot, $1 \mathrm{~mm}$ from the target surface. Ionization of the selected atomic component is accomplished because one of the lasers, referred to here as the resonant laser, is carefully tuned to a resonant frequency of the desired atomic com ponent of the sputtered flux. A second laser, referred to here as the ionizing laser, is of sufficient energy to raise the energy of the resonantly excited atom to a value higher than its ionization potential. The electronically excited atom becomes an ion within a few hundred femtoseconds following absorption of an ionizing photon.

The photoion, once created, can be separated from the secondary ions also produced during the sputtering process on the basis of energy and time. The time discrimination is clear from an examination of fig. 4. The approximately 10 nsec long laser pulse creates the photoions a few hundred nanoseconds after the last secondary ions are produced by the primary ion pulse. The energy discrimination can be understood by examination of fig. 5 . The secondary ions have higher energy than the RIS ions because they are accelerated over the full distance between the sample and lens element 1 (refer to figs. 2 and 5). Photoions on the other hand are created from neutral atoms and molecules only in the laser excitation volume. Figure 5 demonstrates that the potential imparted to photoions created in this region is lower than for the secondary ions. Of course the actual energy of the secondary ions is the sum of their kinetic energy derived in the sputtering process and the potential energy of the applied target voltage. Similarly the energy of the photoions is a function of the sputtered kinetic energy, the distance from the target during laser ionization, and (because lens element 1 has an extraction hole which distorts the potential field) the axial position.

The separation of secondary ions and photoions in the SIRIS apparatus (fig. 2) is accomplished following extraction by a double focusing mass spectrometer. Subsequent detection is accomplished using an ion detector which can oper ate either in a single ion counting mode or in an analog current mode.

Figure 3 depicts the SARISA apparatus [4]. The data collection sequence, which may be understood with reference to fig. 4 (labels 3 ), is initiated when a 0.5 to $2 \mu \mathrm{sec}$ long primary ion pulse enters the energy and angle refocusing time of flight (EARTOF) system on the left. The ion pulse traverses the set of deflection plates and then passes through the primary ion turning plates which merge the primary ions orito the EARTOF axis by means of electrostatic deflection. The beam is then focused onto the target by the high voltage Einzel lens. As can be seen in fig. 4, the primary ion pulse strikes the sample target during a time when the sample potential is held at $1400 \mathrm{~V}$. At $300 \mathrm{~ns}$ following the primary ion pulse, the target potential is lowered to $1100 \mathrm{~V}$ and the two lasers are triggered. Resonant or nonresonant photoionization is used 
to generate positive ions in the spatial region shown in fig. $6-0.5$ to $1.5 \mathrm{~mm}$ from the target surface with a $3 \mathrm{~mm}$ vertical dimension.

Once created the photions are ejected away from the target and into the EARTOF by the large target potential. The photoions traverse the high volt age lens region, and because of the unique lens design [16], the photoions are imaged through the primary ion beam turning plates (which are now at $0 \mathrm{~V}$ ) and onto the entrance arerture of the first resistive disk analyzer. The resis tive disk analyzers are spherical energy analyzers constructed to have large angular and energy acceptance windows. This is accomplished using bound ary electric field matching conditions similar to earlier designs $[14,17]$.

The combination of two spherical analyzers provides two key functions in the SARISA system. First, they strongly suppress all high energy ions giving rise to noise which was produced during the sputtering process and ejected while the target was held at high potential. Second, they serve to reduce the detri mental effects of the large photoion energy spread (fig. 5) on the TOF mass spectrum. Several alternative energy refocusing systems based ori spherical sectors have been designed and constructed $[18,19]$. The inset signal spectrum of fig. 4 depicts a single pulse nonresonant ionization spectrum of atoms sputtered from a Mo metal target in the SARISA apparatus with the 7 naturally occurring isotopes well-resolved. The spectrum was taken with removal of about $10^{6}$ Mo atoms.

\section{Analysis}

Let us first examine the useful yield, $\psi$, for LSNMS techniques. The useful yield may be separated into two parts as follows:

$$
\psi=\psi_{\mathrm{a}} \psi_{\mathrm{ms}}
$$

where $\psi_{\mathrm{ms}}$ is the mass spectrometer transmission factor for photoions created in the laser photoionization region and $\psi_{\mathrm{a}}$ is the fraction of sputtered atoms which are photoionized. $\psi_{a}$ is a function of the laser spot size and position, the laser ionization efficiency, the primary ion pulse width, the relative timing of the laser and ion pulses, the sputtered atom velocity distribution, and for resonant ionization the excited electronic state distribution of the spl'tered flux. Let us examine $\psi_{\mathrm{a}}$ and $\psi_{\mathrm{ms}}$ in turn.

\section{1. $\psi a$}

An understanding of $\psi_{a}$ can be obtained by starting with the simplest case. Figure 6 presents a detailed two-dimensional projection of the photoionization region. Let us first consider $\varepsilon$, the fraction of sputtered atoms in the photoioni zation volume. $\varepsilon$ depends on the number density velocity distribution of sputtered atoms. At any given time, $t_{L}$, following the end of the primary ion pulse there will exist atoms with velocities both too large and too small to be in 
the photoionization volume. An examination of the magnitude of this fraction can be obtained by assuming that the targets of interest sputter as predicted by linear collision cascade theory [20-23]. These theories derive the flux energy distribution of sputtered atoms by assuming that each primary ion impact is independent and produces a collision cascade of target atoms which is isotropic below the surface. This Sigmund-Thompson collision cascade theory has proved remarkably accurate for all measured, sputtered, ground state atom distributions [1,2,24-27]. The approximately $E^{-2}$ energy distribution of colliding atoms is refracted at the surface by a surface binding energy, $E_{b}$, leading to a flux energy distribution of sputtered atoms of

$$
f(w, \theta) d \Omega d w=[\cos (\theta) d \Omega / \pi]\left[2 w d w /(1+w)^{3}\right]
$$

where $w\left(=E / E_{b}\right)$ is a reduced energy parameter, $\theta$ is the angle shown in fig. 6 , and $d \Omega$ is a solid angle element in the direction defined by theta. The angular and energy factors, given individually in square brackets, are normalized to unit integrals. Since the geometry of interest is a small volume element and since we wish to determine the optimum laser firing delay, $t_{L}$, the flux energy distribution must be transformed into the following number density velocity distribution.

$$
g(u) d \Omega d u=\left(\pi / 2 v_{b}\right)[d \Omega / 2 \pi]\left[(16 / \pi)\left(u^{2} d u /\left(1+u^{2}\right)^{3}\right)\right]
$$

where $u\left(=v / v_{b}\right)$ is the reduced velocity parameter and $v_{b}\left(=\left[2 E_{b} / m\right]^{1 / 2}\right)$ can be found from the binding energy. The number density velocity distribution, $g(u)$, is displayed in fig. 7. As a result of the velocity vector dependent flux to number density transformation, $g(u)$ is independent of $\theta$ and peaks at lower $u$ values than does the flux velocity distribution.

For an arbitrarily short primary ion pulse, there exists a one-to-one corre spondence between $g(u)$ and the number density of sputtered atoms along the target normal at any given time, $t$, following the primary ion pulse. There exists a time, $t_{l}$, for which $\varepsilon$ is maximized. The crosshatched region of fig. 7 repre sents the fraction of atoms which are located at a distance between $0.5 \mathrm{~mm}$ and $1.5 \mathrm{~mm}$ from the target along the target normal at $t_{L}$. This represents nearly $60 \%$ of ali the sputtered atoms.

Three conclusions are immediately evident from this analysis. First, a large fraction of ail sputtered atoms are available for photoionization. Second, the optimum delay time between the primary ion pulse striking the target and the laser firing time, $t_{L}$, is different (although with a weak dependence) for each atomic mass. A complete analysis including the three-dimensional laser volume and ion pulses of firite time width is necessary to obtain quantitative numbers for $\varepsilon$. 
In order to analytically examine the two effects on $\varepsilon$ described above, let us return to fig. 6 and assume that the laser volume is a cylinder of height $\left(z_{2}-z_{1}\right)$, and of radius $h$. For this case $g(u)$ can be integrated to find $\varepsilon$ for an arbitrary primary ion pulse width and for any $t_{L}$. The solution is a simple, closed form in two cases - for long and for very short primary ion pulses. The analytical models are useful in that they exhibit parametric dependences explicitly and are available for checking numerically integrated simulations which have been applied to more cornplicated geometries [6]. First consider short ion pulses followed by laser ionization at a time $t_{L}$. In this case

$$
\varepsilon=[\cos (\theta) \mathrm{d} \Omega / \pi]\left[4 \mathrm{u}^{4} /\left(1+\mathrm{u}^{2}\right)^{3} \mathrm{dR} / \mathrm{R}\right]
$$

where $R$ is the distance from the target to the ionization volume center and $R^{2} d R d \Omega$ defines an infinitesimal volume element. For the finite cylindrical volume, eq. 4 can be integrated, using $z=R \cos \theta$ and $R=u v_{b} t_{L}$, to give

$$
\varepsilon=p\left(z_{1}\right)-p\left(z_{2}\right)
$$

where $p(z)=a^{2} /\left(a^{2}+z^{2}\right)-a^{2} /\left(a^{2}+h^{2}+z^{2}\right)$. A feeling for the magnitude of $\varepsilon$ can be found by inserting the parameters for each of the instruments being compared here. For SARISA and SIRIS $z_{1}=0.05 \mathrm{~cm} ; z_{2}=0.15 \mathrm{~cm}$; and $\mathrm{h}=0.15$ $\mathrm{cm}$. In this case $\varepsilon=0.42$ for the optimum value $v_{b} t_{L}=0.075$. For reference $t_{L}=194 \mathrm{~ns}$ for Fe atoms sputtered from an Fe surface. In the case of $S A L I$, this number is reduced to $\varepsilon=0.08$ because of the smaller laser volume employed.

While the shortest primary ion pulses result in the largest c's, the shortest ion pulse is not necessarily the optimum experimental condition. This conundrum arises from the limited duty cycle of laser based experiments. Consider a primary ion pulse of 0.1 picocoulombs. This is clearly close to the delta function limit for pulses of peak currents in the $\mu$ amp range. For materials with a sputtering yield of 1 , there exist only $2.6 \times 10^{5}$ atoms in the SARISA photoionization volume and only $5.2 \times 10^{4}$ in the SALI photoionization volume. In an experiment with a $100 \mathrm{~Hz}$ repetition rate, it would take many seconds to remove even one atom of a ppb impurity.

The necessity of using longer primary ion pulses in order to accomplish impur ity maps in a more reasonable time frame leads us to an examination of the next analytical solution for $\varepsilon$ - the long pulse limit. The fundamental ass! imption made in deriving this equation is that the primary ion pulse is so long that all velocities have uniformly distributed themselves across the photoionization region. In this case the efficiency, in a differential volume element, is

$$
\varepsilon=[\cos (\theta) \mathrm{d} \Omega / \pi]\left[\pi \mathrm{dR} / 4 \mathrm{v}_{\mathrm{b}} \tau\right]
$$


where $\tau$ is the primary ion pulse length. Again, for the finite cylindrical volume of fig. 6 ,

$$
\varepsilon=\left[\pi /\left(2 v_{b} \tau\right)\right]\left[q\left(z_{2}\right)-q\left(z_{1}\right)\right]
$$

where $q(z)=z\left\{1-\left[1+(h / z)^{2}\right]^{1 / 2}\right\}$. Rigorously, the long pulse assumption would require an infinitely long (continuous) primary ion pulse $(\tau \rightarrow \infty)$. Of course the efficiency for this case goes to zero. In practice, however, since the low velocity portion of the sputtered number density velocity distribution (fig. 7) represents a relatively small fraction of the total sputtered number density velocity distribution, a minimum duration can be found for primary ion pulse times, $\tau$, as follows:

$$
\tau=\left\{R_{M} / u^{*}\right\}\left\{1 / v_{b}\right\}
$$

where $u^{*}$ is $v^{*} / v_{b}$. The quartity $v^{*}$ can be defined as the lowest velocity which will traverse the distance $R_{M}$ (fig. 6 ) during $\tau$. The optimum time, $t_{L}$, for firing the laser pulse will typically occur slightly after $\tau$ (so that the volume acquires slightly more slow atr:ms, while escaping fast ones are still being replenished). Thus the assumption of uniformly distributed atom velocities in the photoioni zation volume is rigorously true for all velocities greater than $v^{*}$. For $u^{*}=0.6$, nearly $80 \%$ of all sputtered atoms fall in this range. For $\mathrm{Fe}$ atoms sputtered from an $\mathrm{Fe}$ surface, in the geometry of fig. $5, u^{*}=0.6$ implies $\tau=0.92 \mu \mathrm{s}$, giving $\varepsilon \cong 0.20$. Even in the limit where there were no atoms in the photoionization volume with a velocity less than $v^{*}$, a value of $\varepsilon=0.16$ would be obtained.

It is apparent that in this more complete three-dimensional description, the fraction of photoionizable sputtered atoms decreases somewhat when compared to the simplest one-dimensional case but remains a sizeable fraction of the totel sputtered flux. In fact not all of the atoms which are irradiated in the laser volume become ions. The reason for this is different in the case of resonant ionization and nonresonant ionization. Let us examine the ionization efficiency, $\rho$, for each of these cases in turn.

While, in principle, any atom may be resonantly photoionized, it is useful to consider a particular element, $\mathrm{Fe}$. The energy level diagram of gas phase $\mathrm{Fe}$ atoms is displayed in fig. 8. For resonant ionization two [4] or even three [5] lasers may be used in the photoionization process. In fig. 8 the two-color ionization process is displayed in which ground state $\mathrm{Fe}$ atoms are first resonantly excited using the $y^{5} \mathrm{D}_{4}{ }^{0}-\mathrm{a}^{5} \mathrm{D}_{4}$ transition at $302.065 \mathrm{~nm}$ and then are ionized with a $308 \mathrm{~nm}$ photon. Two-photon ionization of $\mathrm{Fe}$ is a third-order process and thus requires laser intensities in the $10-100 \mathrm{MW} / \mathrm{cm}^{2}$ range to be efficient. However many commercial pulsed lasers are available which can easily supply saturating laser intensities even for the relatively large laserirradiated volumes employed by SARISA and SIRIS. 
Figures 9 and 10 describe SARISA saturation studies of the resonant and ionizing lasers used in the photoionization of $\mathrm{Fe}$ atoms. The studies were conducted using spatially truncated Gaussian laser beams in order to eliminate the effective laser interaction region volume changes normally associated with changes in laser intensity [28]. Furthermore, the multi-step ionization process was accomplished with two separate lasers - a dye laser operating at $302.065 \mathrm{~nm}$ and a $\mathrm{XeCl}$ excimer operating at $308 \mathrm{~nm}$. Independent adjustment of the laser intensities is crucial for careful saturation studies since the transition dipole moment for resonant excitation can be many orders of magnitude larger than for transitions to the ionization continuum. The saturation studies (figs. 9 and 10) demonstrate that effectively all ground state $\mathrm{Fe}$ atoms in the laser volume could be photoionized $(\rho=1)$ with relatively moderate laser powers and that the resonant laser intensity required was nearly one order of magnitude smaller than the ionizing laser intensity. This becomes important since various saturation phenomena such as lifetime broadening and Stark sirifts can actually decrease the photoionization efficiency with increasing intensity [29].

The ground state ionization efficiency of resonant multiphoton ionization can be made to approach unity for riearly every element. However the sputtering process does not leave all the atoms in the ground electronic state. For instance, for $\mathrm{Fe}$ nearly $40 \%$ of the sputtered atoms are in excited states $[1,4,26]$, with most of this population in excited fine structure levels of the ground state. The fraction of excited atoms has been measured for a number of systems $[1,30]$. In all cases the ground state fraction has been $>0.5$. For atoms such as $\mathrm{Li}$ which sputter from high work function matrices with large ion fractions, laser ionization will not be as useful.

The situation for nonresonant multiphoton ionization is somewhat different. Nonresonant ionization accesses all molecules and atoms regardless of excited state with varying efficiencies. The excitation efficiency is largely sample independent and must be calibrated as a function of laser intensity. Figure 11 is a SARISA saturation measurement for $\mathrm{Cu}$ atoms and $\mathrm{Cu}_{2}$ molecules sputtered from a polycrystalline $\mathrm{Cu}$ surface. In this experiment only one nonresonant laser color was used $(308 \mathrm{~nm})$. Clearly saturation is not reached, the fraction ionized being a function of the laser color and intensity. The relatively large laser beam cross sectional area used in SARISA in order to maximize $\varepsilon$ makes saturation difficult to achieve for many atomic and molecular species. Substantially more work on nonresonant MPI has been conducted in the SALI apparatus [3]. In general, it has been found that for $248 \mathrm{~nm}$ light (KrF) saturating intensities can be achieved for most elements in the $10^{8}$ to $10^{11} \mathrm{~W} / \mathrm{cm}^{2}$ intensity range. However, variation of the effective ionization volume with intensity has to be considered in the case of tightly focused laser beams.

Nonresonant excitation will photoionize neutral atoms in all electronic energy levels, Thus to first order, excited state distributions of sputtered atoms are of no concern. However, elements with large secondary ion yields will have effectively lower p's. 
$\Psi$ a can now be found as the product of $\rho$ and $\varepsilon$. For the large laser volumes used in SARISA and SIRIS, it seems possible that $\psi_{a}$ in the range 0.05 to 0.15 can routinely be achieved with resonant excitation. For nonresonant excitation, saturation in many cases will require smaller laser volumes and $\psi_{a}$ values of 0.01 to 0.03 are achievable.

This examination has up until now focused basically upon the possible signal available in LSNMS experiments. Clearly trace analysis requires rejection of large numbers of noise ions in order to achieve meaningful measurements. For resonant ionization experiments, substantial noise rejection is accomplished in the photoionization process. Several noise sources remain including second ary ions of the bulk material, high energy backscattered primary ions and laser-created nonresonant ions. In nonresonant $\mathrm{MPI}$, all the noise rejection must be accomplished in the mass spectrometer with the acivantage, however, that all elements in the sample are detected essentially simultaneously.

\section{2. $\Psi_{\mathrm{ms}}$}

Let us now turn (Iur attention to mass spectrometer transmission functions. Three approaches have been adopted [3-5]. The most straightforward [5] is to interpose a mass filter (either quadrupole of magnetic mass sector) between the photoionization volume and the detector. This results in excellent noise rejection. However, substantial signal loss also results because the transmis sion of ions through these devices can be as poor as $10^{-5}$ and in general their transmission is no better than 10-2. A second method has been to use a TOF mass spectrometer. The mass resolution in many TOF's would be poor because of the energy spread inherent in the extraction process. Consider fig. 5. The atoms in the photoionization region experience a large potentialenergy variation due to differing initial positions in the drawout field. Therefore, two isochronous TOF mass spectrometers have been used.

One apparatus [3] involves the use of a reflectron to reduce the effect of this energy dispersion on the mass resolution of the system. This apparatus has demonstrated an impressive resolution $(\mathrm{m} / \mathrm{sm})$ of 500 . Noise rejection can be a problem in normal reflectrons because of scattered particles from various grids, although gridless designs are now commercially available [31]. One problem with the reflectron approach is that although it is time refocusirig it is not spatially refocusing. This tends to limit the transmission.

A third approzch is that found in SARISA [4]. Spherical energy analyzers when appropriately coupled can produce both energy and angle isochronous refocusing. Several versions of energy refocusing spherical analyzers have been designed and constructed $[18,19]$. Trajectory calculations on the SARISA system (fig. 3) show essentially unit transmission for photoions [32]. This system does not have any grids in the flight path and has a demonstrated mass resolution $(\mathrm{m} / \Delta \mathrm{m})$ of better than 200 .

\section{3. $\psi$, Useful Yield}


A calculation of the possible useful yield in an LSNMS experiment can be made on the basis of the above discussion. For RIS of sputtered $\mathrm{Fe}$ atoms in laser volumes of the order shown in fig. $6\left(z_{1}=0.5 \mathrm{~mm}, z_{2}=1.5 \mathrm{~mm}, \mathrm{~h}=1.5 \mathrm{~mm}\right)$, a $\psi \cong 0.10$ is possible. This should be compared with typical useful yields in SIMS experiments of $10^{-3}$ to $10^{-6}$.

Calculation of a useful yield for nonresonant ionization is somewhat more complicated. It appears that for some atomic species it is possible to achieve $\rho=1$ even for relatively large ionization volumes and thus to reach $\psi=0.10$. However, for most atomic species, smaller laser volumes are required in order to obtain $p=1$. In such cases, $\psi \equiv 0.01$.

\subsection{Sensitivity}

The sensitivity achievable for an impurity constituent for ion bombardment measurements is always a function of the amount of sample consumed. The useful yield is one important parameter in determining the sensitivity achievable for a given sample size. The second important parameter is the noise equivalent signal. That a useful compromise can be drawn for LSNMS considering these two limitations is demonstrated in fig. 12.

Figure 12 maps the Fe concentration of an $56 \mathrm{Fe}$ implanted Si (111) wafer. The ${ }^{56} \mathrm{Fe}$ levels were calibrated by fixing the peak concentration at the $400 \mathrm{ppb}$ level expected for these imp'antation parameters. Similar calibration factors were found by comparison with a SARISA determination of Fe sputtered from a polycrystalline $\mathrm{Fe}$ target. It is assumed that $\mathrm{Fe}$ sputters from the Si substrate with the same yield as $\mathrm{Si}$ from $\mathrm{Si}$ (111) (i. e., $\mathrm{S}=1.4$ ) [33]. It is necessary to include a slight change in the fraction of $\mathrm{Fe}$ atoms in the ground electronic state with changing matiix [4].

The SARISA measurement in fig. 12 demonstrates both a high useful yield and a low-noise equivalent signal. An evaluation of the number of counts detected for $400 \mathrm{ppb} \mathrm{Fe}$ in Si leads to a determination of the total useful yield in SARISA. The useful yield in terms of photoions detected/atoms sputtered was $\psi=0.05$ in this experiment. Since RIS measures only $\mathrm{Fe}$ atoms in the $a^{5} \mathrm{D}_{4}$ state, it is useful to use the known ground state [1] fraction of sputtered $\mathrm{Fe}$ atoms to calculate a $\psi^{\prime}=0.09$ for "ionizable atoms." While the last $56 \mathrm{Fe}$ measurement in fig. 12 reveals an impurity concentration of $8 \pm 2 \mathrm{ppb}$, it is possible also to find the noise equivalent signal from these measurements. This is found by using the useful yield and measured noise counts. For removal of 0.5 monolayers from a $0.25 \mathrm{~mm}$ diameter spot, the noise equivalent signal is found to be $2 \mathrm{ppb}$ for $56 \mathrm{Fe}$ and $500 \mathrm{ppt}$ for $54 \mathrm{Fe}$. The higher value for $56 \mathrm{Fe}$ arises because of background nonresonant ionization of ${ }^{28} \mathrm{Si}_{2}$.

The usefulness of the LSNMS technique in general and the particular type of LSNMS in particular will be dictated by a wide variety of analytical needs and 
sample parameters. For instance the use of nonresonant ionization has already proven particularly useful in studying glasses and more complicated samples [3]. The ability to photoionize all neutral species and the relatively high mass resolution of the reflectron-type apparatus allows examination with very little matrix effects of all the sample constituents. The use of a magnetic mass spectrometer leads to a strongly noise immune system [5]. Measurements based 0 : such devices are very useful for samples with high ion yields, such as insulators [5].

\section{Conclusion}

It seems clear that the high useful yields and relative noise immunity of the LSNMS technique will prove important for understanding many sputtering phenomena and for sensitive, quantitative, surface impurity detection. The wide interest and variety of instruments being tested at the present time are a clear indication of the technique's usefulness. Much work remains to be done, however, to optimize these metinods.

The wide variation in ion fraction and molecular fraction of the sputtering yield as a function of surface chemical environment make it imperative that ion probe instruments be built to accomplish both SIMS and LSNMS during a single measurement. Only by monitoring all the available channels available for sputtered particles can this technique take on a convenient form for routine analysis. 
[1] D.M. Gruen, A.R. Krauss, and M.J. Pellin, Rad. Effects 89 (1985) 113;

D.M. Gruen, M.J. Pellin, C.E. Young, M.H. Mendelsohn, and A.B.

DeWald, Physica Scripta. T6 (1983) 42.

[2] N. Winograd, J.P. Baxter and F.M. Kimock, Chem Phys. Lett. 88 (1982) 581; F.M. Kimock, J.P. Baxter and N. Winograd, Surf. Sci. 124 (1983) L41.

[3] C.H. Becker and K.T. Gillen, Anal. Chem. 56 (1984)1671; Appl. Phys. Lett. 45 (1984) 1063; J. Vac. Sci. Technol. A3 (1985) 1438; in Secondary Ion Mass Spectrometry (SIMS V), eds., A. Bennirighoven, R.J. Colton, D.S. Simons and H.W. Werner (Springer, Berlin, 1986) p.85.

[4] M. J. Pellin, C. E. Young, W. F. Calaway, J. W. Burnett, B. Jørgensen, E. L. Schweitzer, and D. M. Gruen, Nucl. Instr. and Meth. B18 (1987) 446; C. E. Young, M. J. Pellin, W. F. Calaway, B. Jørgensen, E. L. Schweitzer and D. M. Gruen, Resonance lonization Spectroscopy, eds., G. S. Hurst et al. (Inst. Phys. Conf. Ser. No. 00, Bristol, 1986) p 000; M. J. Pellin, C. E. Young, W. F. Calaway and D. M. Gruen, Nucl. Instr. and Meth. B13 (1986) 653; Surf. Sci. 144 (1984) 619.

[5] J. E. Parks, H. W. Schmitt, G. S. Hurst and W. M. Fairbanks Jr., Thin Solid Films 8 (1983) 69; J. E. Parks, D. W. Beekman, L. J. Moore, H. W. Schmitt, M. T. Spaar, E. H. Taylor, J. M. R. Hutchinson and W. M. Fairbank, Jr., Resonance Ionization Spectroscopy, eds., G. S. Hurst, et al. (Inst. Phys. Conf. Ser. No. 00, Bristol, 1986) p. 000; 11th International Congress on X-ray Optics and Microanalysis, London, Ontario, Canada, Aug. 4-8, 1986.

[6] F. M. Kimock, J. P. Baxier, D. L. Pappas, P. H. Kobrin, and N. Winograd, Anal. Chem. 56 (1984) 2782; F. M. Kimock, D. L. Pappas and N. Winograd, Anal. Chem. 57 (1985) 2669. 
[7] W. H. Christie, D. E. Goeringer, Resonance lonization Spectroscopy (Inst. Phys. Conf. Ser. No. 00, Bristol, 1986), p. 000, eds. G. S. Hurst, et al.

[8] A. R. Krauss and R. B. Wright, J. Nucl. Mater. 89 (1984) 229.

[9] M. J. Pellin, C. E. Young, D. M. Gruen, Y. Aratono, and A. B. DeWald, Surf. Sci. 151 (1985) 477; M. J. Pellin, C. E. Young, M. H. Mendelsohn, D. M. Gruen, R. B. Wright and A. B. DeWald J. Nucl. Mater. 111/112 (1982) 738.

[10] W. Husinsky, P.Wurz, B.Strehl, and G. Betz, Nucl. Instr. Methods B18 (1987) 452.

[11] R. N. Zare, Science 226 (1984) 2980.

[12] H. Oechsner, W. Rühe and E. Stumpe, Surf. Sci. 85 (1979) 289.

[13] W. Reuter, in: Secondary Ion Mass Spectrometry (SIMS V); eds., A. Benninghoven, R.J. Colton, D.S. Simons, and H.W. Werner (Springer, Berlin, 1986) p. 94.

[14] D. Lipinsky, R. Jede, O. Ganschow and A. Benninghoven, J. Vac. Sci. Technol. A3 (1985) 2007.

[15] B. A. Mamyrin, V. I. Karataev, D. V. Shmikk and V. A. Zagulin, Sov. Phys. - JETP 37 (1973) 45.

[16] H. Liebl, Int. J. Mass Spectrom. Ion Phys. 46 (1983) 511.

[17] M. W. Siegel and M. J. Vasile, Rev. Sci. Instr. 52 (1981) 1603.

[18] W. P. Poschenrieder, Int. J. Mass Spectrom. Ion Phys. 9 (1972) 357.

[19] T. Sakurai, T. Matsuo and H. Matsuda, Int. J. Mass Spectrom. Ion Phys. 63 (1985) 273.

[20] M. W. Thompson, Philos. Mag. 18 (1968) 377.

[21] P. Sigmund, Phys. Rev. 184 (1969) 383.

[22] P. Sigmund, Rev. Roum. Phys. 17 (1972) 769, 897, 1005. 
[23] P. Sigmund, in: Inelastic lon-Surface Collisions, eds., N. H. Tolk, J. C. Tully, W. Heiland, and C. W. White (Academic Press, New York, 1977) p. 121.

[24] D. Hammer, E. Benes, P. Blum, and W. Husinsky, Rev. Sci. Instrum. 47 (1976) 1178; W. Husinsky, R. Bruckmüller, P. Blum, F. Viehböck, D. Hammer, and E. Benes, J. Appl. Phys. 48 (1977) 4734; P. Blum in: Proc. $7^{\text {th }}$ Int. Vacuum Congress and 3rd Int. Conf. on Solid Surfaces, eds., R. Dobroozemsky, F. Rudenauer, F. P. Viehböck, A. Breth (Institut für Allgemeine Physik, TU Wien 1977) p 1469; W. Husinsky, R. Bruckmüller, P. Blum, Nucl. Instrum. Meth. 170 (1980) 287; W. Husinsky, G. Betz, and I. Girgis, Phys. Rev. Lett. 50 (1983)1689; J. Vac. Sci. Technol. A2 (1984) 698; W. Husinsky, G. Betz, 1. Girgis, F. Viehböck, H. L. Bay, J. Nucl. Mater. 128/129 (1984) 577; W. Husinsky, I. Girgis, G. Betz, J. Vac. Sci. Technol. B3 (1985) 1543.

[25] W. Husinsky and G. Betz, Nucl. Instrum. Meth. B15 (1986) 165.

[26] B. Schweer, H. L. Bay in: Proc. IVth Int. Conf. on Solid Surface Science, and IIIrd Europ. Conf. on Surface Science, eds., D. A. Degras, M. Costa (Societe Francaise du Vide, Paris, 1980) p. 1349; E. Hintz, D. Rusbuldt, B. Schweer, J. Bohdansky, J. Roth, and A. P. Martinelli, J. Nucl. Mater. 93/94 (1980) 656; B. Schweer, H. L. Bay, Appl. Phys. A29 (1982) 53; H. L. Bay, W. Berres, E. Hintz, Nucl. Instr. ana Meth. 194 (1982) 555; E. Dullni, Appl. Phys. A38 (1985) 131; H.L. Bay, Nucl. Instr. and Meth. B18 (1987) 430.

[27] R. B. Wright, M. J. Pellin, D. M. Gruen and C. E. Young, Nucl. Instr. and Meth. 170 (1980) 295; R. B. Wright, M. J. Pellin and D. M. Gruen, Nucl. Instr. and Meth. 182/183 (1981) 167; Surf. Sci. 110 (1981) 151; M. J. Pellin, R. B. Wright and D. M. Gruen, J. Chem. Phys. 74 (1981) 6448; R. 
B. Wright, C. E. Young, M. J. Pellin and D. M. Gruen, J. Vac. Sci. Tech. 20 (1982) 510; C. E. Young, W. F. Calaway, M. J. Pellin, D. M. Gruen, J. Vac. Sci Tech. A2 (1984) 693

[28] M. B. Blackburn, J. M. Mermet, G. D. Boutilier and J. D. Winefordmer, Appl. Opt. 18 (1979) 1809.

[29] L. Allen, R. W. Boyd, J. Krazinski, M. S. Malcuit and C. R. Stroud, Jr., Phys. Rev. Lett. 54 (1985) 309.

[30] M. J. Pellin, D. M. Gruen, C. E. Young and M. D. Wiggins, Nucl. Instr. and Meth. 218 (1983) 771.

[31] Bruker Analytische Messtechnik GMBH.

[32] B. Jorgensen, M. J. Pellin, W. F. Calaway, C. E. Young, E. L. Schweitzer, J. W. Burnett and D. M. Gruen, this conference.

[33] H. H. Andersen and H. L. Bay in: Topics in Applied Physics, Vol. 47, ed., R. Benrisch (Springer, Berlin, 1981) p.145. 


\section{Figures}

1. A schematic diagram of the nonresonant laser ionization instrument of Becker and Gillen [3].

2. A schematic diagram of the sputter-initiated resonance ionization spectrometer (SIRIS) system [5]

3. The SARISA III Energy and Angle Refocusing Time-of-Flight (EARTOF) system [4] for detection of sputtered neutral atoms. The element labels are self explanatory. A detailed description of the instrument operation may be found in the text.

4. A LSNMS timing diagram. The labels 1, 2, and 3 refer to functions which are specific for the apparatus described in references 3,5 and 4, respectively.

5. The graph depicts the idealized potential between two plates of infinite extent. Superimposed onto the graph is a diagram of the photoion extraction region for an LSNMS instrument. Clearly, the potential energy of the secondary ions which are created at the target $(+V)$ is larger than that of the photions created between the plates.

6. Detail of the laser ionization region. $z_{1}$ and $z_{2}$ define the axial extent of the laser beams, $\theta$ is the target normal referenced ejection angle of the sputtered atom, and $\mathrm{h}$ the largest off-axis position from which photions 
may be collected by tr a detection optics. The $1 \mathrm{~mm}$ distance is simply to provide the reader with a typical scaling factor.

7. Depicted is the number density velocity distribution of atoms sputtered with a Sigmund-Thompson [20-23] distribution. In the limit of a delta function primary ion pulse, there exists a one-to-one correspondence of points on this curve and the number density of atoms at a given distance from the target. The quantity $u$ represents the reduced velocity $\left(=v / v_{b}\right)$. The crosshatched region represents the fraction of atoms which are between 0.5 and $1.5 \mathrm{~mm}$ from the target for a delta function primary ion pulse at a time $t_{L}$.

8. An $\mathrm{Fe}$ atom energy level diagram showing the transitions used for resoriant ionization of sputtered Fe atoms.

9. $\mathrm{Fe}^{+}$signal as a function of resonant laser intensity for a SARISA measurement of $\mathrm{Fe}$ atoms sputtered from a Si matrix. Note that saturation is achieved for moderate laser intensities.

10. Fe+ signal as a function of ionizing laser intensity for a SARISA measurement of $\mathrm{Fe}$ atoms sputtered from a Si matrix. Note that saturation of the ionization laser signal requires substantially higher laser intensities than for the resonant step.

11. Intensity dependence of the SARISA Cu+ and $\mathrm{Cu}_{2}^{+}$photoion signal for nonresonant multiphoton ionization with $308 \mathrm{~nm}$ light. The $\mathrm{Cu}$ atoms 
and molecules were sputtered by $3.7 \mathrm{keV} \mathrm{Ar}^{+}$primary ions from a polycrystalline Cu surface.

12. SARISA depth profile of $56 \mathrm{Fe}$ implanted Si (111). A $60 \mathrm{keV}, 10^{11}$ atoms $/ \mathrm{cm}^{2}$ implantation dose was used to prepare the sample. The $56 \mathrm{Fe}$ and $54 \mathrm{Fe}$ detection limits are the calculated impurity limits for a signal-to-noise ratio of 1 . Each measurement is made with removal of 0.5 of a monolayer. 


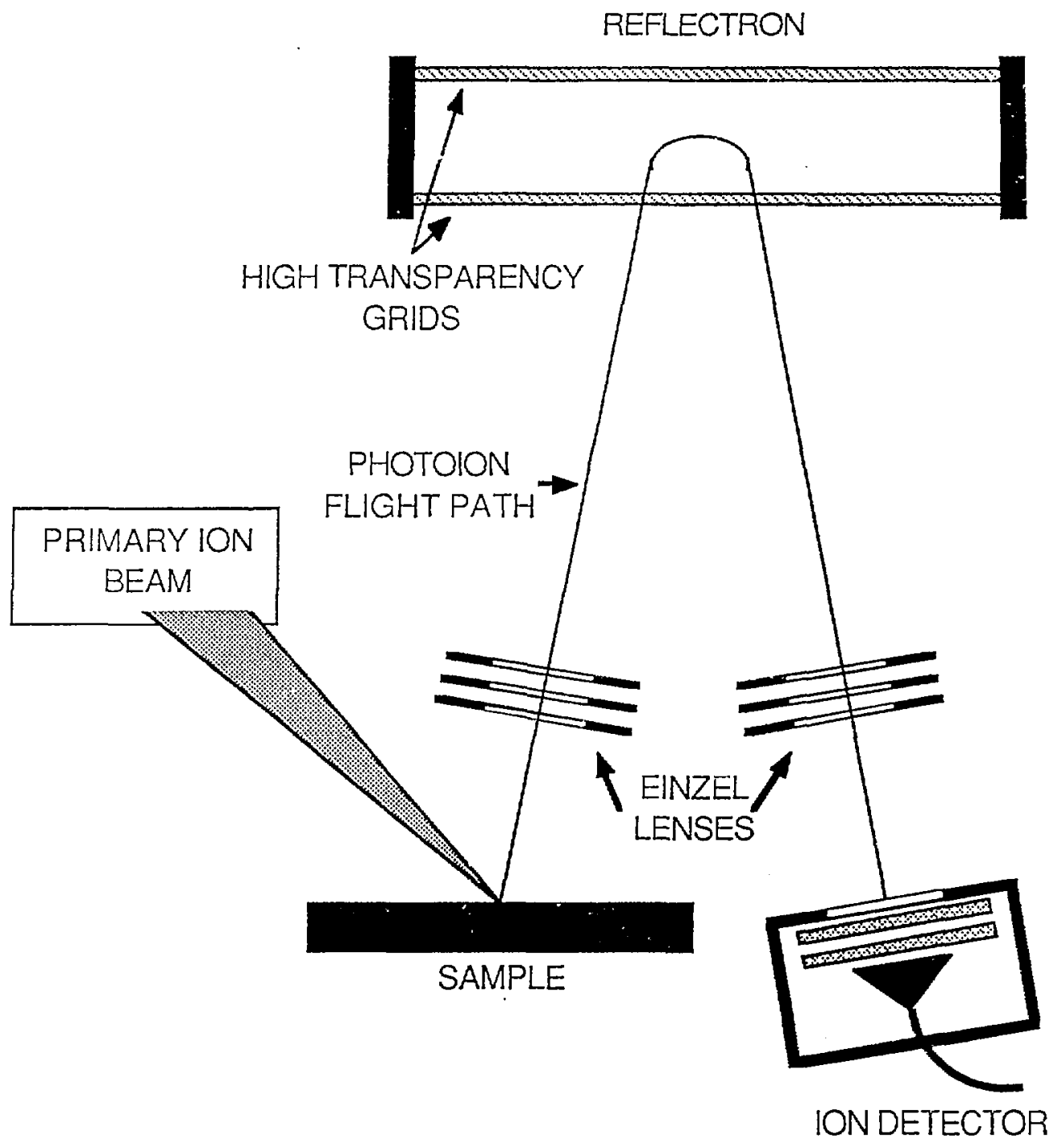


DOUBLEFOCUSING

MASS SPECTROMETER

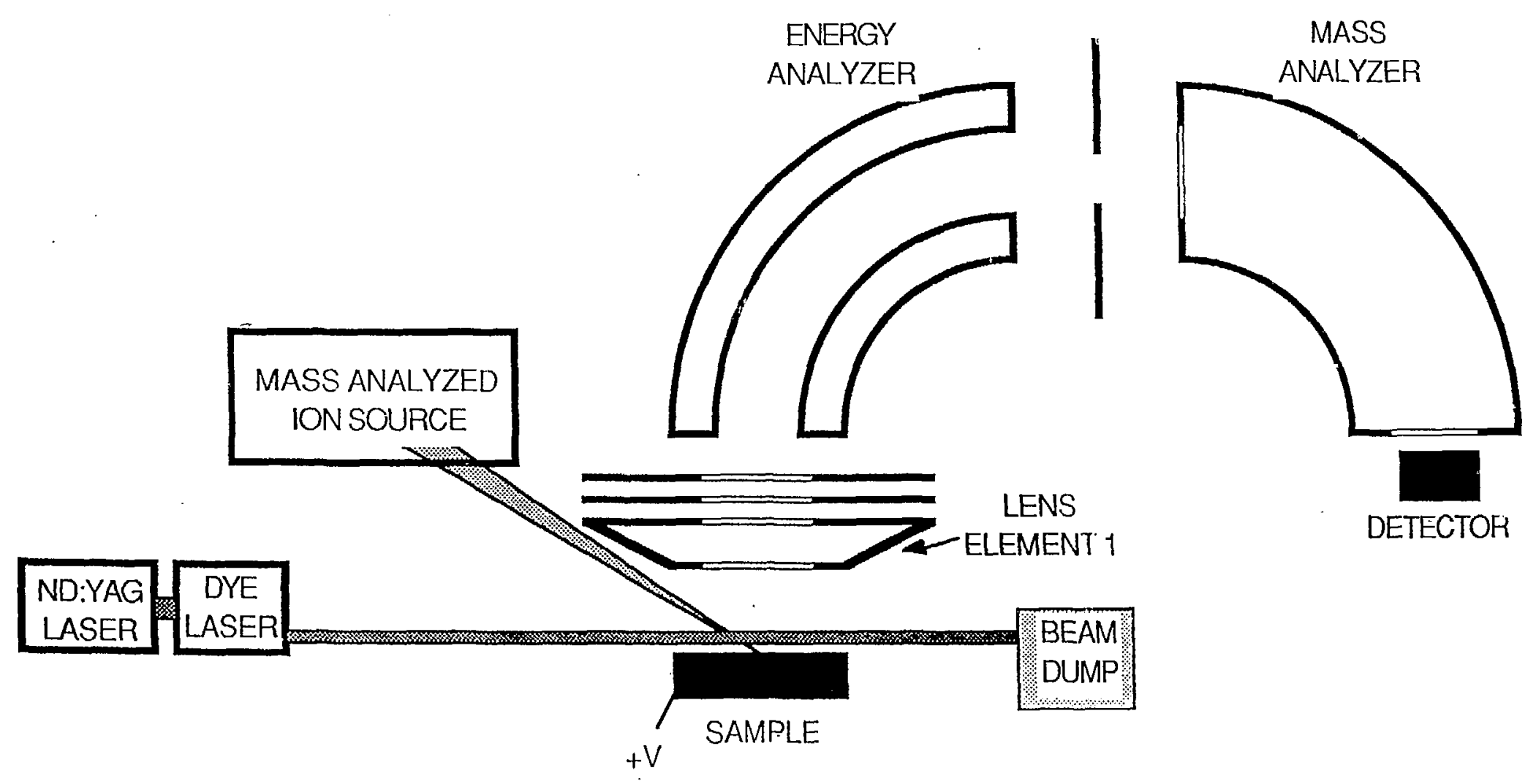




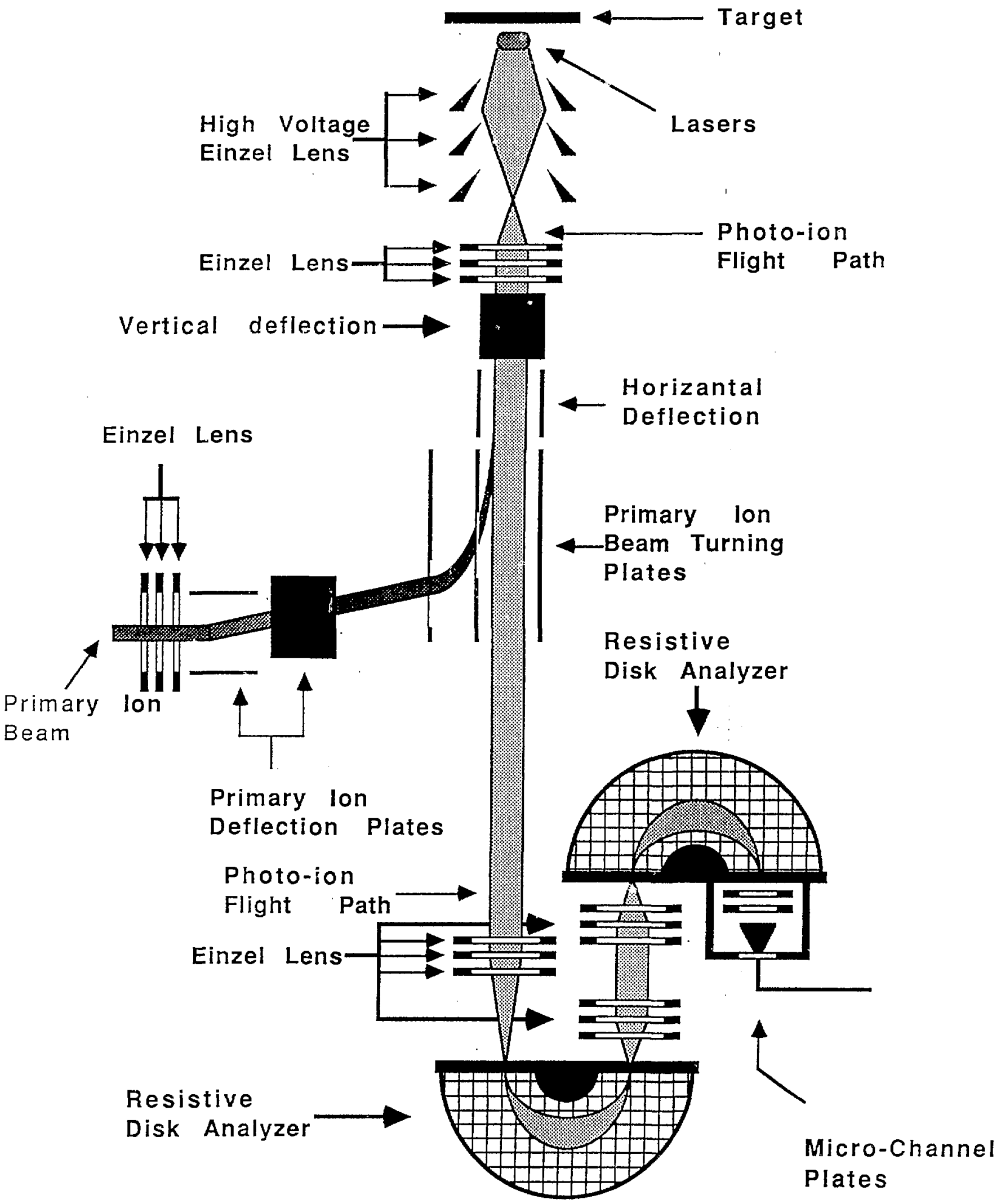




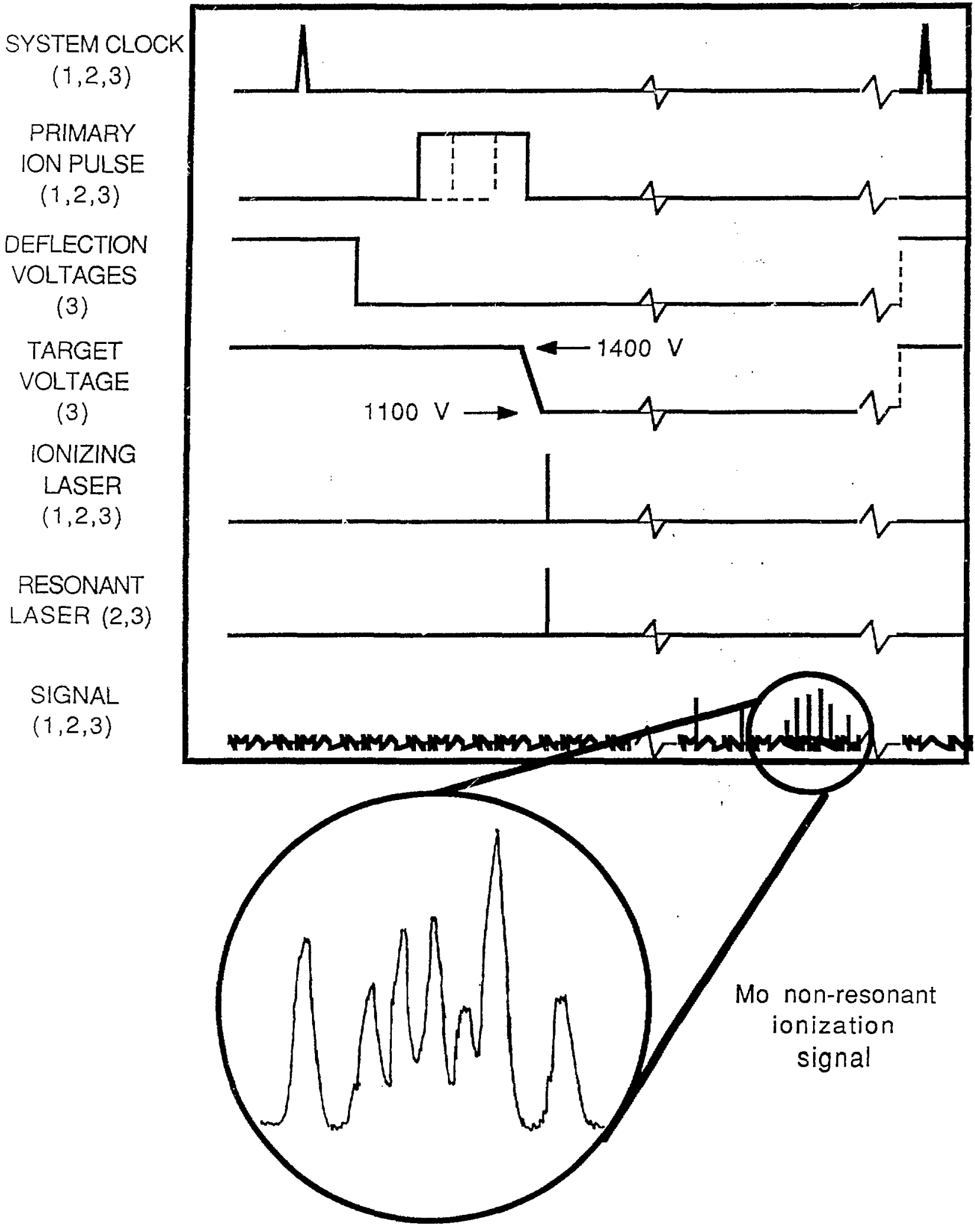




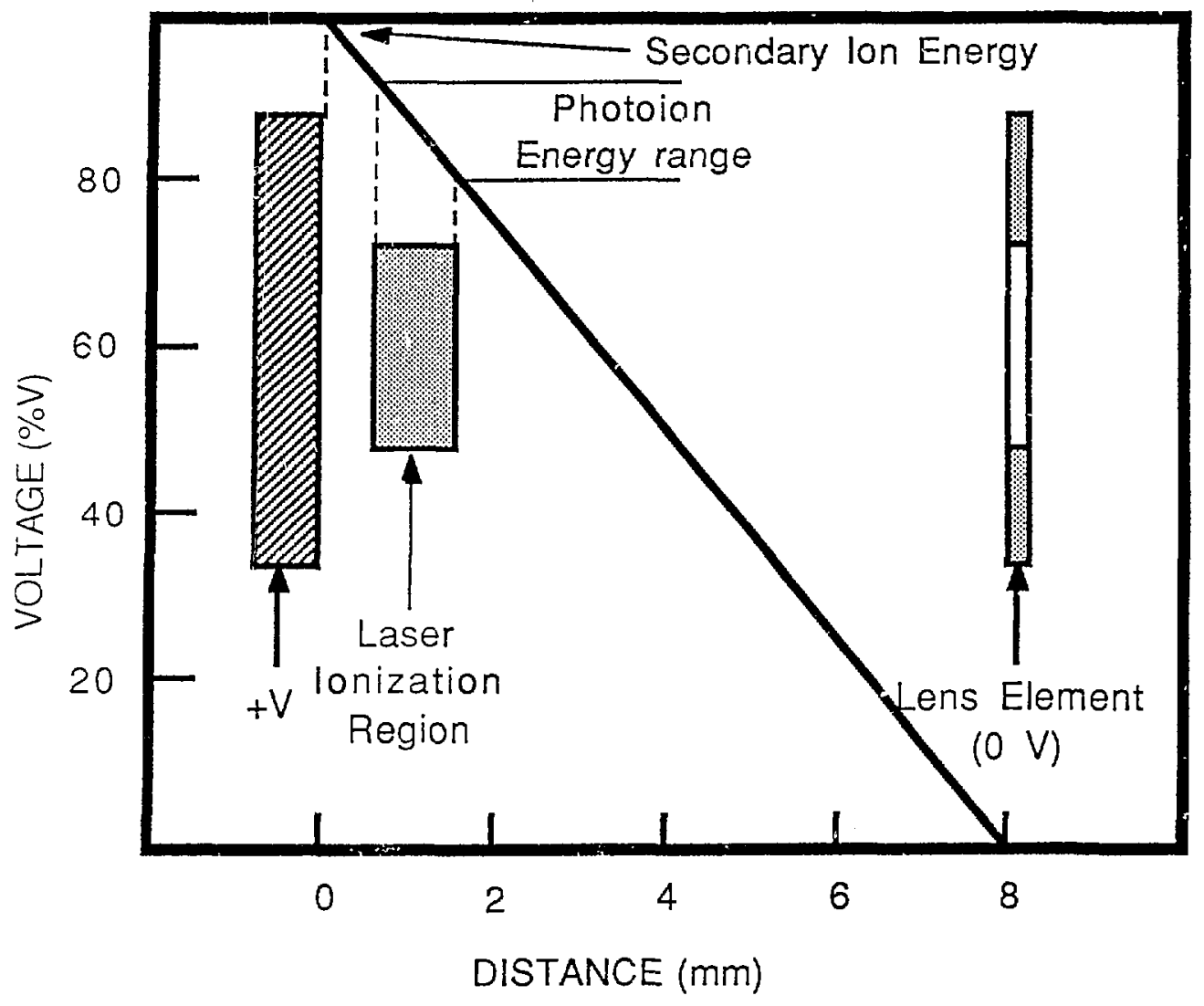




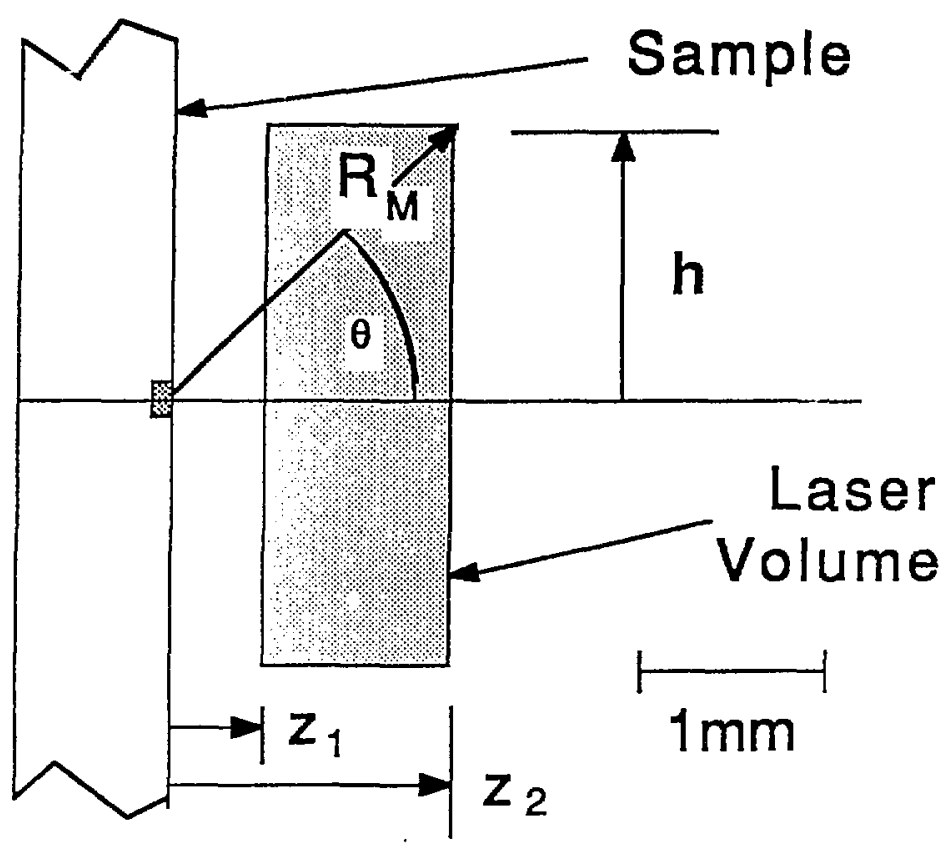




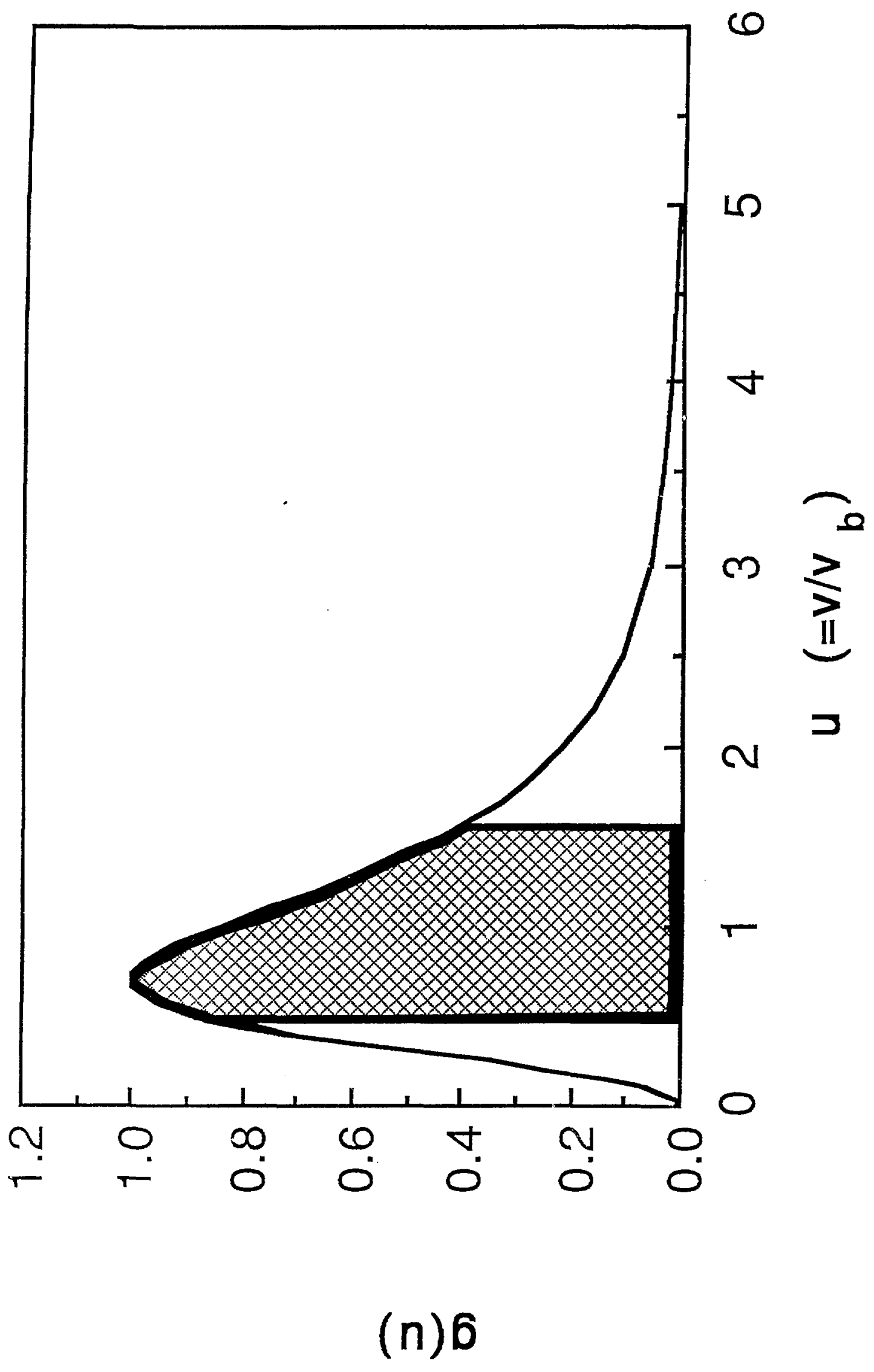


$\mathrm{Fe}$

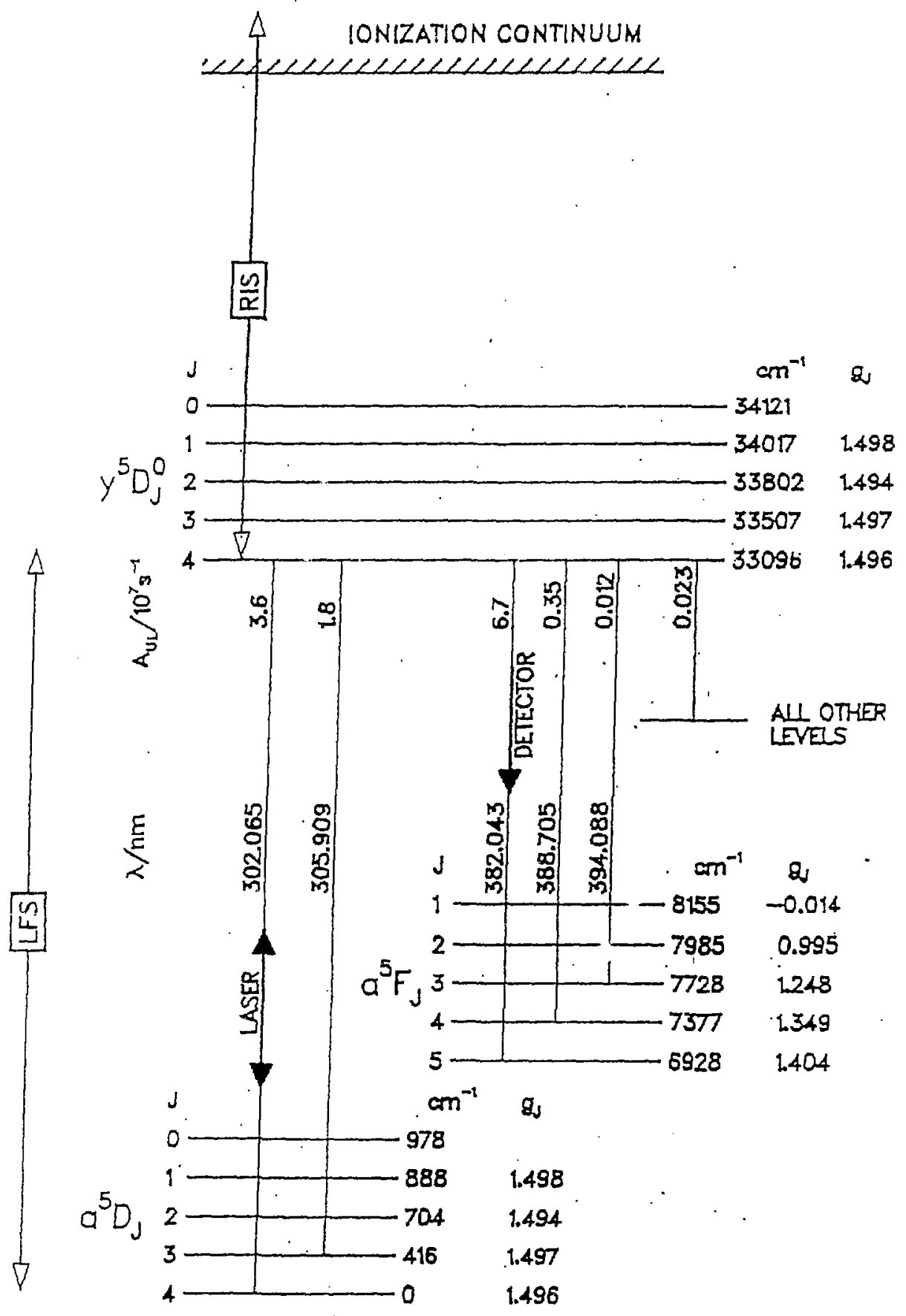




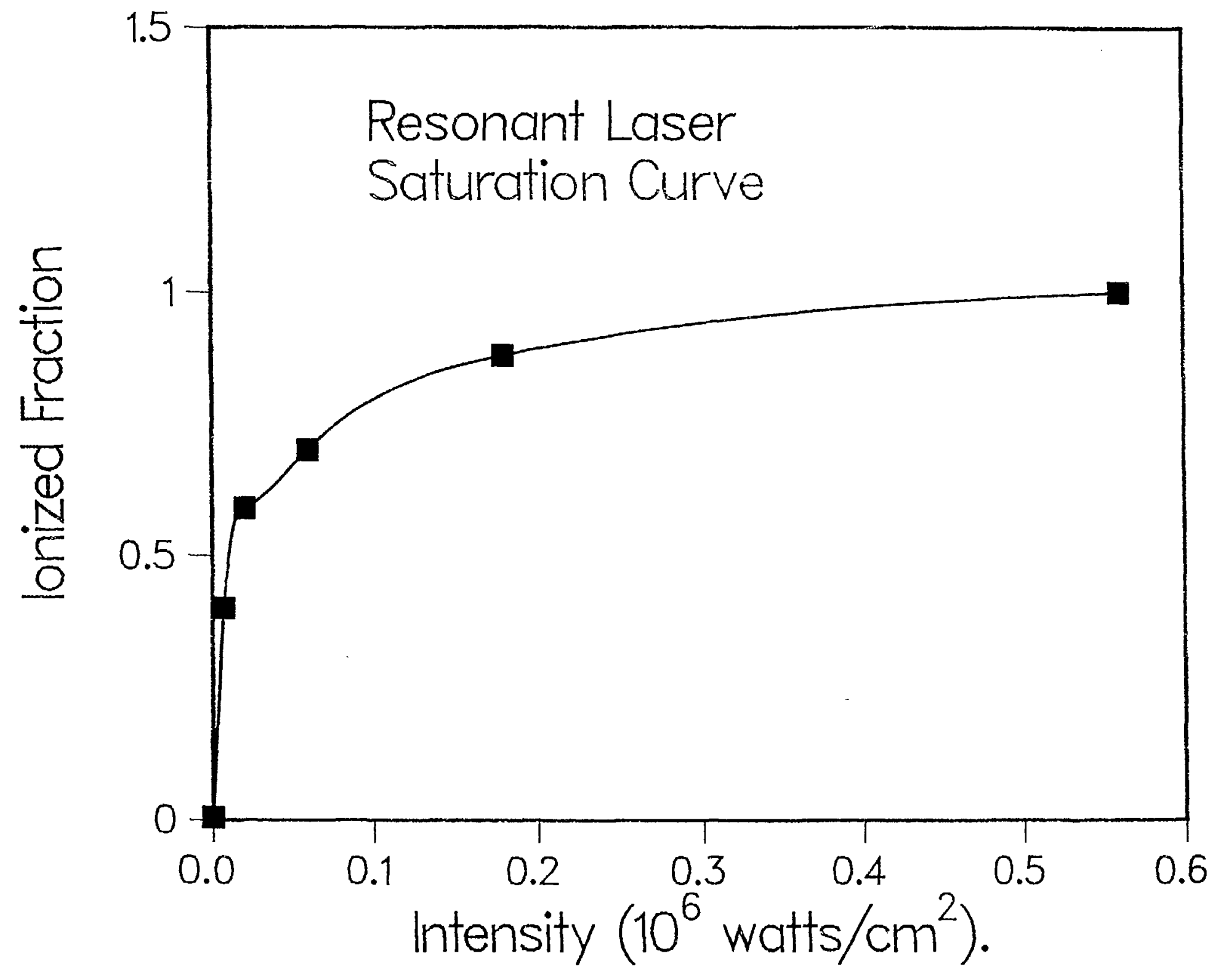




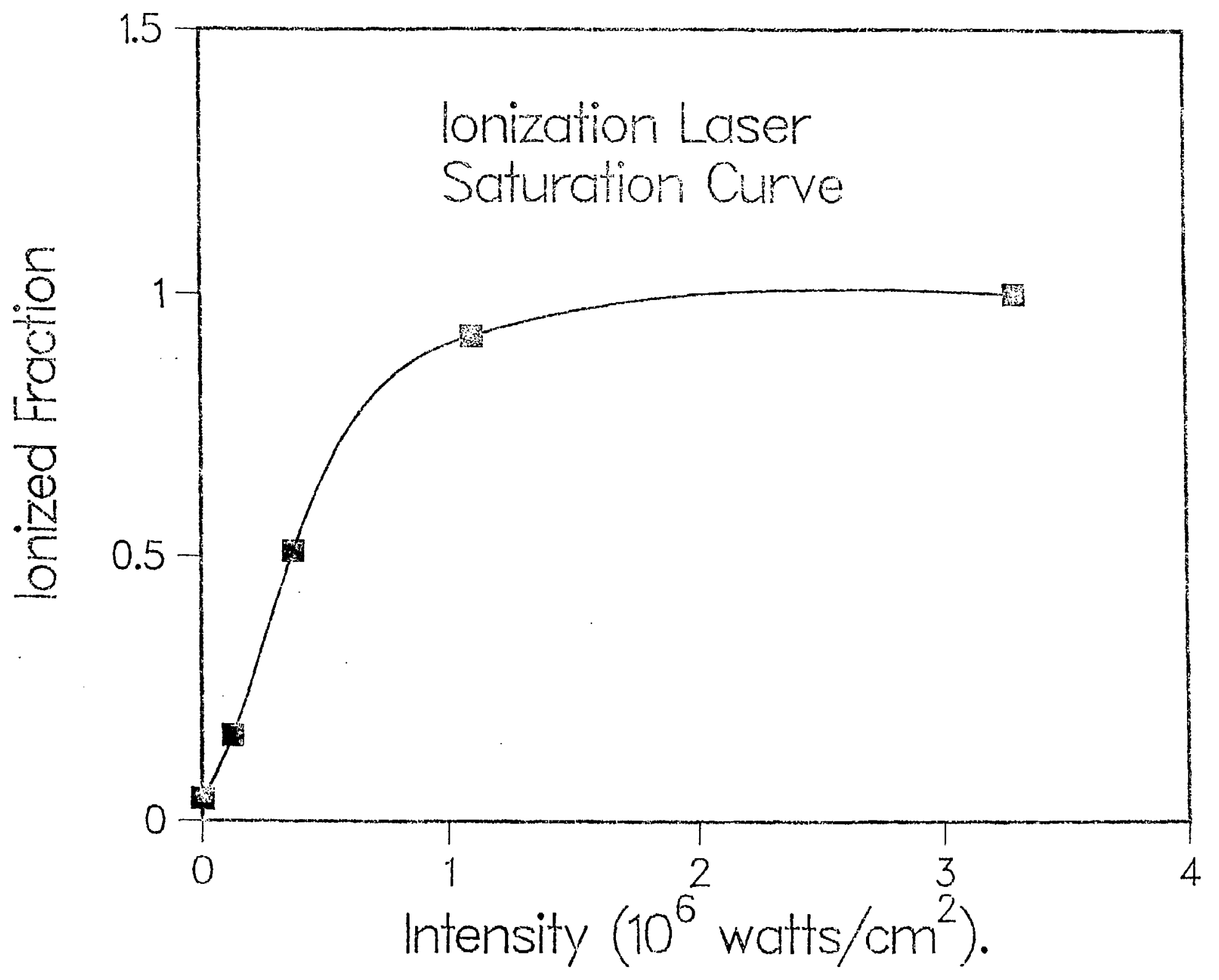




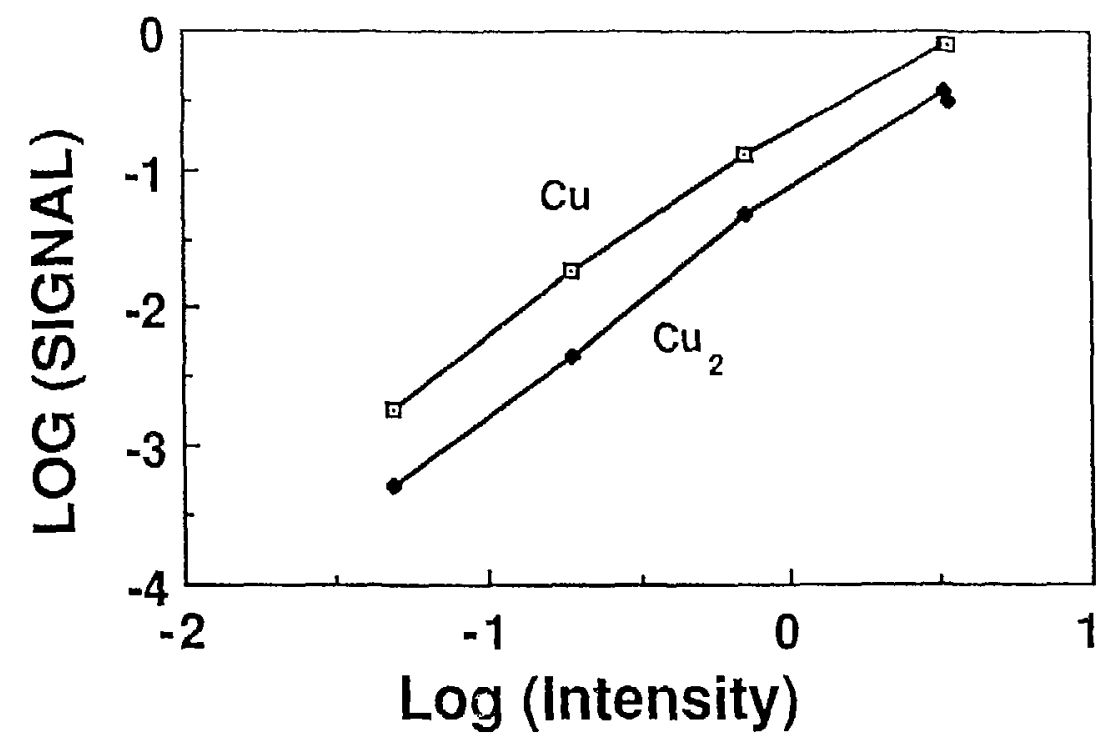




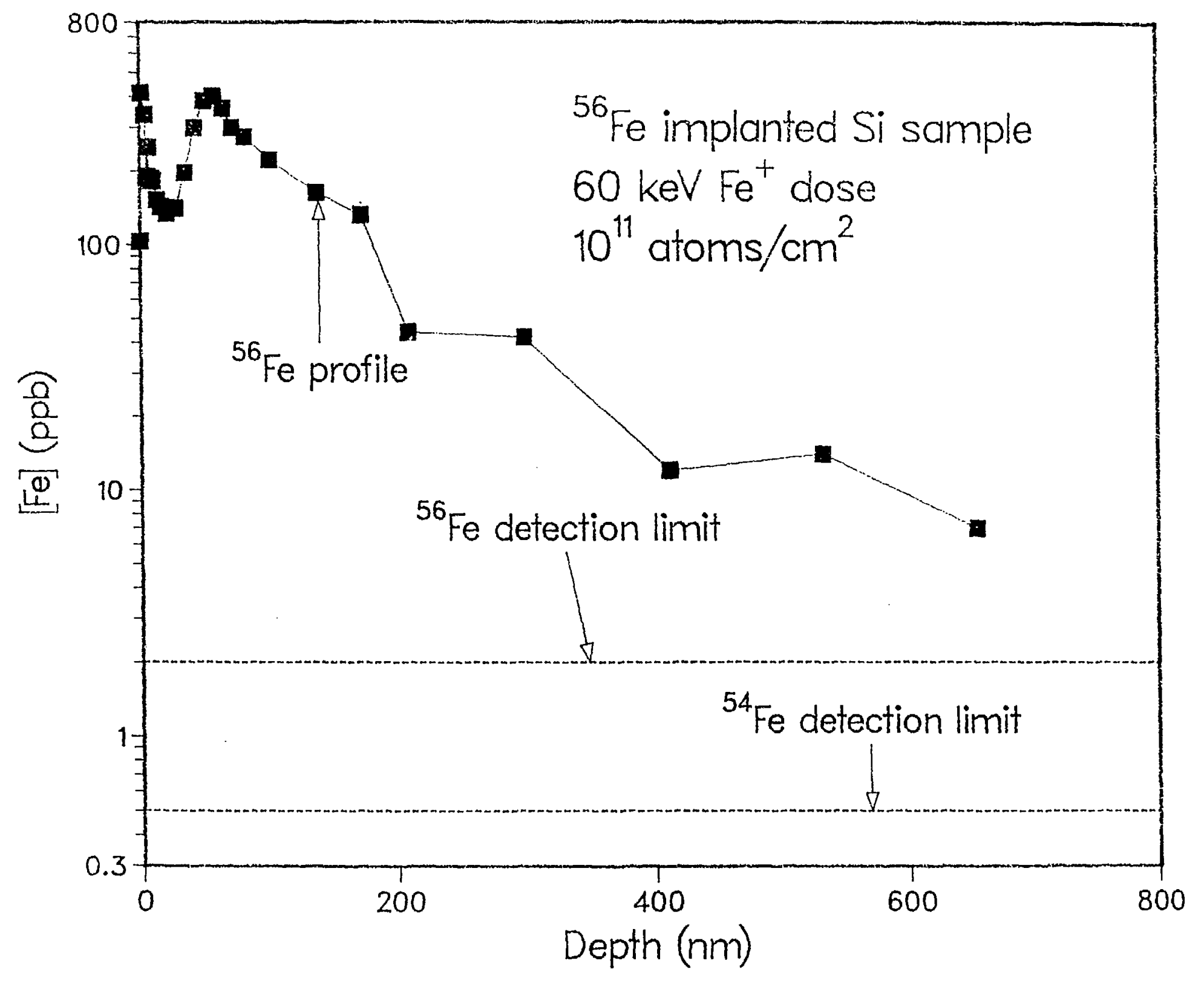

